\title{
Avaliação das necessidades de treinamento - uma metassíntese
}

\author{
Evaluation of training needs: a meta-synthesis
}

\author{
Angélica Maria da Silva ${ }^{1}$ \\ Carolina Machado Saraiva de Albuquerque Maranhão² \\ Talita Almeida Fernandes ${ }^{3}$
}

\section{Resumo}

A elaboração e implementação de um programa de treinamento e desenvolvimento de recursos humanos que permita a flexibilização das organizações frente às constantes exigências dos ambientes interno e externo são alguns dos principais objetivos das organizações que buscam a sua sobrevivência e manutenção de seu capital intelectual. Para tal, é necessário saber identificar as necessidades de treinamento na empresa. Desenvolver esse diagnóstico não é tarefa simples. Tal tema tem sido alvo de estudos acadêmicos e mercadológicos, buscando estruturar um sistema de Análise de Necessidades de Treinamento (ANT). Uma análise desses modelos propostos revela uma dissonância entre os estudiosos. A fim de alcançar esse objetivo, foi realizada uma metassíntese segundo os critérios estabelecidos por Hoon (2013). A identificação dos modelos se deu por meio de uma pesquisa bibliométrica nos anais dos eventos EnANPAD e EnGPR dos anos de 2003 a 2014, bem como nas edições do mesmo período dos periódicos classificados no espectro A1-B2, considerados os de maior impacto pelo sistema Qualis Capes. Acredita-se que esta pesquisa contribui para a compreensão da área de gestão de pessoas como estratégica nas organizações, através da sistematização de seus processos e otimização dos seus indicadores de resultados.

Palavras-chave: Análise de necessidades de treinamento. Treinamento. Competências.

1 Bacharel em Administração pela Universidade Federal de Ouro Preto. Endereço de contato: Rua do Catete, 285 apt. 1, Centro, Mariana-MG. Telefone: (31) 8883-8257 - Brasil - E-mail: angelicams16@yahoo.com.br

2 Doutora em Administração pela UFMG e Professora Adjunta da Graduação em Administração da Universidade Federal de Ouro Preto. Endereço de contato: Rua Santo Agostinho, 567 apt 1001. Bairro Sagrada Família. Belo Horizonte-MG CEP: 31.035-480. Telefone: (31) 8482-4166 Brasil - E-mail: carola.maranhao@gmail.com

3 Graduanda em Administração pela Universidade Federal de Ouro Preto. Endereço de contato: Rua Hamilton Lázaro da Silva, 26. Bauxita. Ouro Preto-MG. Telefone: (31) 9345-7838 - Brasil E-mail: talitaafernandes16@gmail.com 


\section{Abstract}

The design and implementation of a training program and development of human resources that allow the flexibility of organizations to the requirements of the internal and external environment are some of the main objectives of organizations seeking their survival and maintenance of its intellectual capital. To do this, you must know how to identify training needs in the company. Developing this diagnosis is not an easy task. This topic has been the subject of academic and market studies, seeking to structure a training needs analysis system (TNA). An analysis of these proposed models reveal a dissonance among scholars. In order to accomplish this, we performed a meta-synthesis according to the criteria established by Hoon (2013). The identification of the models was made through a bibliometric research in conference proceedings EnANPAD and EnGPR the years 2003-2014, as well as in issues of the same period of the journals of the A1-B2 spectrum, considered the greatest impact by Qualis Capes system. We believe that this research contributes to the understanding of management as a strategic area of people in organizations through the systematization of processes and optimization of its performance indicators.

Keywords: Analysis of training needs. Training. Skills.

\section{Introdução}

O contexto de valorização e reconhecimento do potencial humano considera que os indivíduos são dotados de inteligência e potencial que precisam ser incentivados, e o colaborador, na perspectiva do capital humano, deve estar inserido em um contexto fundamentado por políticas específicas e assertivas de gestão - políticas estas que compõem o subsistema de treinamento e desenvolvimento. Pozas e Jauregui (2012) afirmam que as empresas acreditam que a utilização adequada e eficaz dos processos de treinamento e desenvolvimento torna-se crucial para o crescimento e sucesso de seu negócio. As autoras definem treinamento e desenvolvimento como um conjunto que compreende atividades planejadas por uma empresa para melhorar as capacidades, habilidades e comportamentos dos funcionários. O treinamento adequado otimiza a relação indivíduo-organização, propiciando melhor desempenho das tarefas e eficiência estratégica. 
De acordo com Pilati (2006), o processo de Treinamento, Desenvolvimento e Educação (TD\&E) proporciona o aprimoramento das competências humanas fundamentais para a continuidade, permanência e crescimento das organizações. Esse processo tem se tornado cada vez mais estratégico, fazendo-se necessária a criação de uma cultura de participação e o diagnóstico das necessidades de treinamento e desenvolvimento, para que assim as organizações possam alcançar suas metas.

Para Pozas e Jauregui (2012), a finalidade desse processo é a preparação dos funcionários para novos desafios e oportunidades em seu local de trabalho, desenvolvendo conhecimentos, habilidades e comportamentos adequados.

Portanto, a elaboração e implementação de um programa de treinamento e desenvolvimento de recursos humanos que permita a flexibilização das organizações frente às constantes exigências do ambiente interno e externo são alguns dos principais objetivos das organizações que buscam a sua sobrevivência e manutenção de seu capital intelectual. Para tal, é necessário saber identificar as necessidades de treinamento na empresa. Após esse diagnóstico, são elaboradas as estratégias e os planos de desenvolvimento do capital intelectual das organizações.

Contudo, geralmente, as organizações não têm a ciência de sua real necessidade de treinamento nos âmbitos motivacionais, comportamentais, estruturais, de habilidades, competências, entre outros. Desenvolver esse diagnóstico não é tarefa simples. Tal tema tem sido alvo de estudos acadêmicos e mercadológicos, buscando estruturar um sistema de Análise de Necessidades de Treinamento (ANT) ou Levantamento das Necessidades de Treinamento (LNT). Esses esforços acadêmicos se concentram nas áreas de administração e psicologia, principalmente. Cada estudo busca estruturar um modelo de LNT, a fim de sinalizar às organizações as áreas e funções de maiores demandas, qual foco de cada treinamento a ser desenvolvido e até mesmo quais as particularidades de cada função em relação aos aspectos comportamentais de seus executores. 
Uma análise desses modelos propostos revela uma dissonância entre os estudiosos. Diferentes critérios são elencados, diferentes propostas são apresentadas, gerando ainda mais desestrutura para o campo, que busca o inverso: a determinação de um modelo válido de análise das necessidades de treinamento de uma organização; um modelo que se aplique ao máximo de casos possível e que seja prescritivo, embasando as decisões estratégicas da área de gestão de pessoas. Da forma como se apresentam, os modelos propostos pelos estudos não compõem um corpus coeso, dificultando a assertividade da tomada de decisões estratégicas.

Buscando sistematizar o conhecimento construído sobre esse tema, propõe-se neste trabalho analisar os modelos de LNT presentes na literatura acadêmica e estruturar os achados sob os seguintes critérios: escopo, objetivos, características, vantagens e desvantagens, bem como as implicações gerenciais de cada modelo analisado. Esse esforço de sistematização é etapa fundamental para a consolidação de um conhecimento científico sobre o tema.

A fim de alcançar esse objetivo, foi realizada uma metassíntese segundo os critérios estabelecidos por Hoon (2013). A identificação dos modelos deu-se por meio de uma pesquisa bibliométrica nos anais dos eventos EnANPAD e EnGPR dos anos de 2003 a 2014, bem como nas edições do mesmo período dos periódicos classificados no espectro A1B2, considerados os de maior impacto pelo sistema Qualis Capes.

Acredita-se que esta pesquisa contribui para a compreensão da área de gestão de pessoas como estratégica nas organizações, por meio da sistematização de seus processos e otimização dos seus indicadores de resultados. A importância do estudo do tema de LNT é ressaltada, com o intuito de que, a partir desse diagnóstico, seja possível implementar programas de treinamento direcionados às reais necessidades dos colaboradores e organizações. 


\section{Necessidades de Treinamento}

Segundo Gil (2001), desde meados da década de 1980, desafios organizacionais, ambientais e globais exigiram a melhoria do desempenho organizacional. Para se adaptarem, as empresas adotaram uma nova forma de gestão de recursos humanos, designada Gestão de Pessoas. Gil (2001, p. 17) define Gestão de Pessoas como sendo "uma função gerencial que visa à cooperação das pessoas que atuam nas organizações para o alcance dos objetivos tanto organizacionais, quanto individuais". Afirma também que "Gestão de Pessoas" tem por objetivo substituir a expressão "Administração de Recursos Humanos".

A mudança no conceito da área de Recursos Humanos para Gestão de Pessoas é inerente ao novo papel estratégico a ser desempenhado, buscando integrar os objetivos de longo prazo da organização, as variáveis relevantes do ambiente e as necessidades do capital humano.

A Gestão de Pessoas possui como subsistemas a seleção, avaliação, recompensa e desenvolvimento, a partir de um enfoque estratégico e, principalmente, a partir da visão de que a organização deve estabelecer uma relação recíproca com seus colaboradores. Essa visão estratégica supera o caráter instrumental, para uma atuação política, social, ideológica e comportamental.

Dessa maneira, a política de Gestão de Pessoas busca a manutenção dos indivíduos na organização de modo flexível, favorecendo a atuação das equipes, com foco no desempenho e alcance das estratégias organizacionais.

Os novos paradigmas organizacionais impõem novas premissas de produção e organização do trabalho e uma cultura de constante aperfeiçoamento e qualificação profissional. A fim de acompanhar esse ritmo de mudanças e obter ganhos mais efetivos em suas ações, as organizações passam a investir na estruturação de sistemas de Treinamento Desenvolvimento e Educação (TD\&E), articulados com o cenário estratégico (ABBAD; MOURÃO, 2012; BORGES-ANDRADE). 
A área de Treinamento e Desenvolvimento de pessoas é um instrumento essencial na formulação de respostas ágeis e flexíveis ao novo cenário e exigências organizacionais. Meneses (2007) esclarece que um sistema de TD\&E abarca três subsistemas que são definidos como campos diferenciados de conhecimento. Para o autor, o subsistema de Análise de Necessidade de Treinamento (ANT) ou Levantamento de Necessidades de Treinamento (LNT) contempla a investigação de condições e fatores organizacionais e ambientais que podem influenciar as demandas por novos conhecimentos, habilidades e atitudes, necessários ao bom desempenho no trabalho. O segundo subsistema, Planejamento e Execução Instrucional, busca o estabelecimento de objetivos instrucionais e escolhas quanto aos métodos e técnicas de treinamentos. E o último subsistema, Avaliação de Efeitos de TD\&E, compreende um processo sistematizado de coleta de informações para avaliação das ações realizadas e retroalimentação do sistema, com foco em sua melhoria constante.

As ações de Treinamento e Desenvolvimento devem sempre estar alinhadas às estratégias da organização. Essa determinação ocorre na Avaliação das Necessidades de Treinamento, sendo identificadas também as competências que precisam ser desenvolvidas em cada pessoa através de treinamentos.

O diagnóstico de necessidades de treinamento possui como base a compreensão sobre o conceito de competência. Para Fleury e Fleury (2001), competência compreende a capacidade se saber agir de forma responsável, promovendo a mobilização, integração, transferência de conhecimentos, recursos e habilidades, que agregam valor econômico à organização e valor social ao indivíduo.

Ferreira (2009) afirma que, apesar do recorrente discurso sobre a necessidade do alinhamento entre as ações de Treinamento, Desenvolvimento e Educação (TD\&E) às estratégias e aos resultados para os grupos, equipes e organização como um todo, a Análise de Necessidades de Treinamento (ANT) não tem sido realizada de modo sistemático em ambientes organizacionais. 
Pilati (2006) já ressaltava à época que a produção nacional encontra-se praticamente estagnada, com permanência de dificuldades na articulação de necessidades de desempenho de indivíduos, grupos e equipes aos objetivos organizacionais, devido à maior ênfase dada aos estudos voltados para análise nos níveis de tarefas e individual. Acreditase que essa desarticulação ainda existe, baseada na metassíntese que se realiza nesta pesquisa.

De acordo com Silva (2010), na literatura nacional, ainda há carência de abordagens proativas em ANT com foco no contexto e nas estratégias organizacionais. Para a autora, o que se verifica são práticas reativas, assistemáticas e informais, pouco alinhadas aos objetivos estratégicos das organizações. Desse modo, durante a análise das necessidades de treinamento, devem-se considerar os contextos interno e externo em que a organização encontra-se inserida, para assim caracterizar o tipo de influência que o ambiente exerce sobre os indivíduos.

Para Meneses, Zerbini e Abbad (2010), é fundamental a compreensão das discrepâncias entre os desempenhos reais, manifestados pelos indivíduos, e o esperado pelas empresas para aproximar as ações de treinamento dos objetivos organizacionais. $O$ conhecimento sobre essas discrepâncias aumenta a probabilidade de sucessos dos programas de treinamento. Dessa forma, deve-se ressaltar que o diagnóstico das necessidades de treinamento deve ser realizado de forma minuciosa, sendo apoiado em fatos concretamente apurados.

A estipulação das necessidades de treinamento é considerada indispensável, porque, além das próprias razões da organização, está inerente aos fatores externos, como o mercado de trabalho, desenvolvimento econômico e tecnológico. Desse modo, uma análise das necessidades de treinamento realizada de maneira apropriada garante aumento na credibilidade do treinamento e objetivos organizacionais, principalmente quando envolve a alta administração.

A principal estrutura utilizada para se diagnosticar a necessidade de treinamento é divida em análise organizacional, análise de tarefas e análise individual, conforme detalhadas a seguir. 
A análise organizacional refere-se ao estudo holístico da organização, tendo como principal objetivo determinar quais as formas e os lugares necessários para se aplicar treinamentos dentro da organização. Segundo Meneses, Zerbini e Abbad (2010), a análise organizacional é responsável pelo alinhamento das ações de TD\&E com a estratégia organizacional. De acordo com Gil (1994), uma boa análise organizacional possibilita identificar como ocorre o crescimento da organização, a que se deve, quais os fatores que o dificultam, e verifica em que medida os recursos humanos disponíveis vêm contribuindo para o alcance dos objetivos da organização. Para o autor, esse tipo de análise possibilita o diagnóstico de algumas necessidades de treinamento, mediante obtenção de dados que constituem indicadores de necessidades.

Considerando-se a visão de Moraes (2002, p. 94), "a Análise de Tarefa identifica a natureza das tarefas a serem executadas no trabalho e os conhecimentos, habilidades, aptidões e atitudes necessárias para o desempenho das mesmas", ou seja, os CHA. Essa análise possui como objetivo a tarefa, levando em consideração as características que um indivíduo deve possuir para ser capaz de executá-la. Corroborando, Meneses, Zerbini e Abbad (2010) consideram que as ações de TD\&E geram efeitos sobre o desempenho das unidades organizacionais, recomendando que a identificação dos CHA focalize os processos de trabalho executados nas unidades selecionadas. O nível de análise de tarefa procura identificar e detalhar as atribuições e responsabilidades do papel ocupacional; para isso, algumas estratégias e procedimentos são utilizados, como análise documental, observação participativa, entrevista, incidentes críticos, entre outros.

Para Meneses, Zerbini e Abbad (2010), a análise individual consiste em identificar quais pessoas devem ser treinadas e os tipos de treinamentos adequados para cada uma delas. Desse modo, essa análise é necessária, pois busca determinar questões relacionadas a conhecimentos, habilidades e atitudes, as quais devem ser desenvolvidas para se obter um bom desempenho no treinamento e, consequentemente, na execução das atividades no trabalho. Um 
instrumento utilizado durante a análise individual é o cálculo de Índice de Prioridade de Treinamento, que consiste em estabelecer indivíduos e competências com maiores prioridades de treinamento.

\section{Metodologia}

A pesquisa cientifica é um instrumento essencial na busca do conhecimento. Segundo Cervo, Bervian e Silva (2007, p. 7), "o conhecimento científico vai além do empírico, procurando compreender, além do ente, do objeto, do fato e do fenômeno, sua estrutura, sua organização e funcionamento, sua composição, suas causas e leis". A ciência é entendida como uma procura constante de explicação e revisão de seus resultados. Nessa busca rigorosa, a ciência pretende aproximarse cada vez mais da verdade através de métodos que estabeleçam maior controle e sistematização, buscando renovar-se e reavaliar-se continuamente. Dessa maneira, Santos (2007, p.17) considera que "a pesquisa científica pode ser caracterizada como atividade intelectual intencional que visa a responder às necessidades humanas".

Esta pesquisa, de cunho qualitativo, é do tipo exploratória e descritiva. Foi utilizado o modelo de metassíntese de Hoon (2013) para a condução da pesquisa. Essa metodologia tem como característica a análise dos diversos casos qualitativos relativos ao tema estudado, em busca de uma consolidação. Essa consolidação não se restringe ao ajuntamento de dados, pois inclui a análise dos casos particulares, seguido da categorização de cada caso e a busca de parâmetros entre o corpus analisado, gerando a metassíntese. Adiante, seguem as etapas previstas por Hoon (2013) para a condução de pesquisas com esse objetivo:

- Enquadramento da questão de pesquisa

- Localização de uma pesquisa relevante

- Critérios de inclusão e exclusão

- Extração e codificação dos dados

- Análise específica de cada caso

- Síntese em um nível de estudo cruzado 
- Construção da teoria a partir da metassíntese

- Discussão

A etapa bibliométrica foi realizada nos anais de eventos EnANPAD e EnGPR dos anos de 2003 a 2014, bem como nas edições do mesmo período dos periódicos classificados no espectro A1-B2, considerados os de maior impacto pelo sistema Qualis Capes. As palavras-chave utilizadas para a seleção amostral foram "levantamento de necessidades de treinamento", "LNT" e "análise de necessidades de treinamento".

O primeiro conjunto amostral foi composto por quinze artigos. Procedeu-se à leitura a fim de se estabelecerem aqueles que seriam excluídos. O critério de exclusão/inclusão foi de que o artigo deveria apresentar um modelo de LNT. Pesquisas de estudo de caso, relatos de experiências ou levantamentos bibliográficos foram excluídos. Finda essa etapa, o corpus continha cinco artigos. A listagem dos artigos analisados encontra-se no Apêndice A.

\section{Análise dos dados}

\subsection{Análise específica de cada caso}

\section{a. Modelo de Abbad e Mourão (2012)}

O modelo proposto é um diagnóstico de necessidades de treinamento baseado em taxonomia de resultados de aprendizagem e considerando diversos níveis de análise. O principal foco é a identificação de necessidades relacionadas ao alcance de resultados e objetivos estratégicos. As autoras tomaram como base o modelo apresentado por Birdi (2006) para avaliação de treinamento e desenvolveram outro modelo específico para a análise de necessidades de TD\&E.

As competências a serem desenvolvidas nos indivíduos são pertencentes a qualquer um dos três domínios da aprendizagem, denominados cognitivo, psicomotor e afetivo, apresentado na teoria das taxonomias de aprendizagem de Bloom et al. (1972). Entretanto, as autoras não explicam no artigo a diferença desses domínios e do que 
realmente se trata essa taxonomia, dificultando o entendimento sobre a relação entre as competências e os domínios de aprendizagem.

Durante a análise desse modelo, pode-se constatar que o aspecto sociedade que já é destacado no artigo deve passar a ser tratado como nível, a fim de abarcar uma maior abrangência em relação aos fatores influenciadores das necessidades de treinamento. Desse modo, o modelo não possui nove etapas, e sim quatro níveis (sociedade, organização, grupo/equipe e indivíduo), os quais devem ser relacionados com os demais fatores (processos, afetivo, cognitivo estratégias, cognitivo estratégias/habilidades e físico-psicomotor).

O modelo de ANT proposto vai além da análise de tarefas e pessoas, incluindo também o nível organizacional e de grupo, permitindo fornecer as informações necessárias ao desenho de situações de aprendizagem e treinamento que possam promover o desempenho de complexas competências, valorizadas pelo mundo do trabalho. Cabe ressaltar, também, a necessidade de que esse modelo teórico seja testado empiricamente para confirmar os níveis e as relações propostas.

\section{b. Modelo de Silva e Meneses (2012)}

Este estudo objetivou, mediante execução de análise de covariância em amostra de 213 participantes, investigar a relação entre motivação para o trabalho e complexidade de necessidades de treinamento, com vistas a permitir a composição futura de modelos teóricos de análise de necessidades integrados, não apenas por componentes relacionados às tarefas, mas também por variáveis relativas aos níveis individual, grupal e organizacional de análise.

Foram mapeadas três categorias, identificadas como adequadas para compor o questionário: leis e regulamentos; normas e procedimentos internos; e visão sistêmica. Assim, foram identificadas 34 descrições resultantes dessa pesquisa documental relacionadas a essas três categorias.

O modelo possui os tópicos "Método de pesquisa" e "Resultados e discussão", que tratam sobre a criação do questionário e demais 
objetivos da pesquisa. A compreensão dos textos abordados nesses tópicos não é totalmente clara, pois muitas vezes um assunto que deveria ser tratado somente em um dos tópicos é abordado nos dois ou em outro, dificultando um melhor entendimento sobre os métodos e resultados referentes a cada etapa.

Foi considerada a importância de se ponderar os níveis de complexidade de aprendizagem estabelecidos pela taxonomia de Bloom et al. (1972), porém, os autores não explicam do que realmente se trata e como de deu essa divisão que abarca os seguintes níveis de aprendizagem: conhecimento, compreensão, aplicação, análise, síntese e avaliação.

A elaboração e validação semântica e estatística desse instrumento de ANT foi uma importante contribuição desta pesquisa, sendo necessário enfatizar, portanto, que o conteúdo do instrumento sobre visão sistêmica é pertinente a qualquer organização, podendo ser facilmente adaptado e aplicado em pesquisas futuras ou na prática.

\section{c. Modelo Castro e Borges-Andrade (2004)}

A terceira análise, referente ao modelo "Identificação das necessidades de capacitação profissional: o caso dos assistentes administrativos da Universidade de Brasília", destinava-se à construção de instrumental de Levantamento de Necessidades de Treinamento (LNT), à aplicação dos questionários construídos e à análise dos resultados.

Foi desenvolvida uma tecnologia de averiguação de necessidades de treinamento, permitindo verificar os resultados do Índice de Prioridade de Treinamento (IPT) caso a caso, tendo-se melhores parâmetros para a tomada de decisão. Dessa forma, o IPT, apesar de não ser o único, mostrou-se ser um bom norteador para a tomada de decisões dos Recursos Humanos, com aplicabilidade prática do conhecimento científico na gestão de programas de Treinamento \& Desenvolvimento e na realidade de vida e do trabalho. 
Portanto, para Castro e Borges-Andrade (2004), esse estudo, além de ser o maior e mais abrangente trabalho de Levantamento de Necessidades de Treinamento realizado até então pela Universidade de Brasília, privilegiou o vínculo acadêmico e o suporte científico aos trabalhos de diagnóstico das necessidades de capacitação profissional, oferecendo subsídios para se ter uma melhor compreensão da clientela e dos relevantes trabalhos que precisam ser realizados na UnB em aspectos que dizem respeito ao treinamento dos assistentes administrativos e suas reais necessidades no trabalho.

\section{d. Modelo Ribeiro, Abbad, Mourão e Meneses (2012)}

O estudo realizado por Ribeiro, Abbad, Mourão e Meneses (2012), exposto no artigo "Análise de necessidades de treinamento para a carreira de especialistas em políticas públicas", tem como objetivo geral analisar necessidades de treinamento para a carreira de Especialista em Políticas Públicas e Gestão Governamental (EPPGG). Cabe ressaltar que o estudo justifica-se em função da relevância que o tema assume no pouco explorado campo teórico-empírico de ANT e no próprio contexto da gestão pública, em processo de reformulação dessa carreira exclusiva.

Os fatores mais recorrentes que supostamente originam e influenciam necessidades de capacitação dos EPPGG enquadram-se na categoria "ambiente organizacional". Em seguida, os EPPGG apontaram algumas especificidades organizacionais como fatores originários ou influenciadores de necessidades de capacitação e, por fim, com menor frequência, as características da própria carreira dos EPPGG.

\section{e. Modelo Silva e Rowe (2012)}

A pesquisa realizada por Silva e Rowe (2012), exposta no artigo "Análise das necessidades de treinamento: um estudo da área de gestão da Fiocruz na Bahia", tem como objetivo geral identificar o nível de alinhamento entre a estratégia organizacional e as ações de TD\&E promovidas pelo Centro de Pesquisas Gonçalo Moniz, unidade da Fiocruz 
na Bahia. Por conseguinte, apresentam-se os objetivos específicos: realizar análise do contexto interno e externo a organização; identificar as competências emergentes; levantar as ações de treinamento no corte temporal 2010 e 2011; identificar o nível de alinhamento entre a estratégia organizacional e as ações de TD\&E.

Esse modelo foi escolhido por melhor atender parte dos objetivos da pesquisa, considerando aspectos do ambiente interno e externo à organização e necessidades de treinamento, apresentando os níveis de análise, macro (organizacional), meso (tarefas, grupos e macroprocessos) e micro (indivíduo), além de ampliar a metodologia do papel ocupacional, por considerar variáveis de contexto e contemplar diferentes níveis de análise do processo de ANT e de necessidades de treinamento, classificando também as necessidades de acordo com a taxonomia de resultados de aprendizagem.

O estudo apresentou clareza na exposição de suas etapas, sendo cada uma explicada em seu momento e com os resultados separados em tópicos, facilitando a compreensão. Cabe ressaltar também que esse estudo alcançou todos os objetivos propostos, obtendo relevância nos âmbitos social, institucional e acadêmico.

\subsection{Síntese em um nível cruzado}

No Quadro 1, apresenta-se a etapa da síntese referente aos modelos estudados, a fim de analisá-los, comparando suas semelhanças e diferenças: 


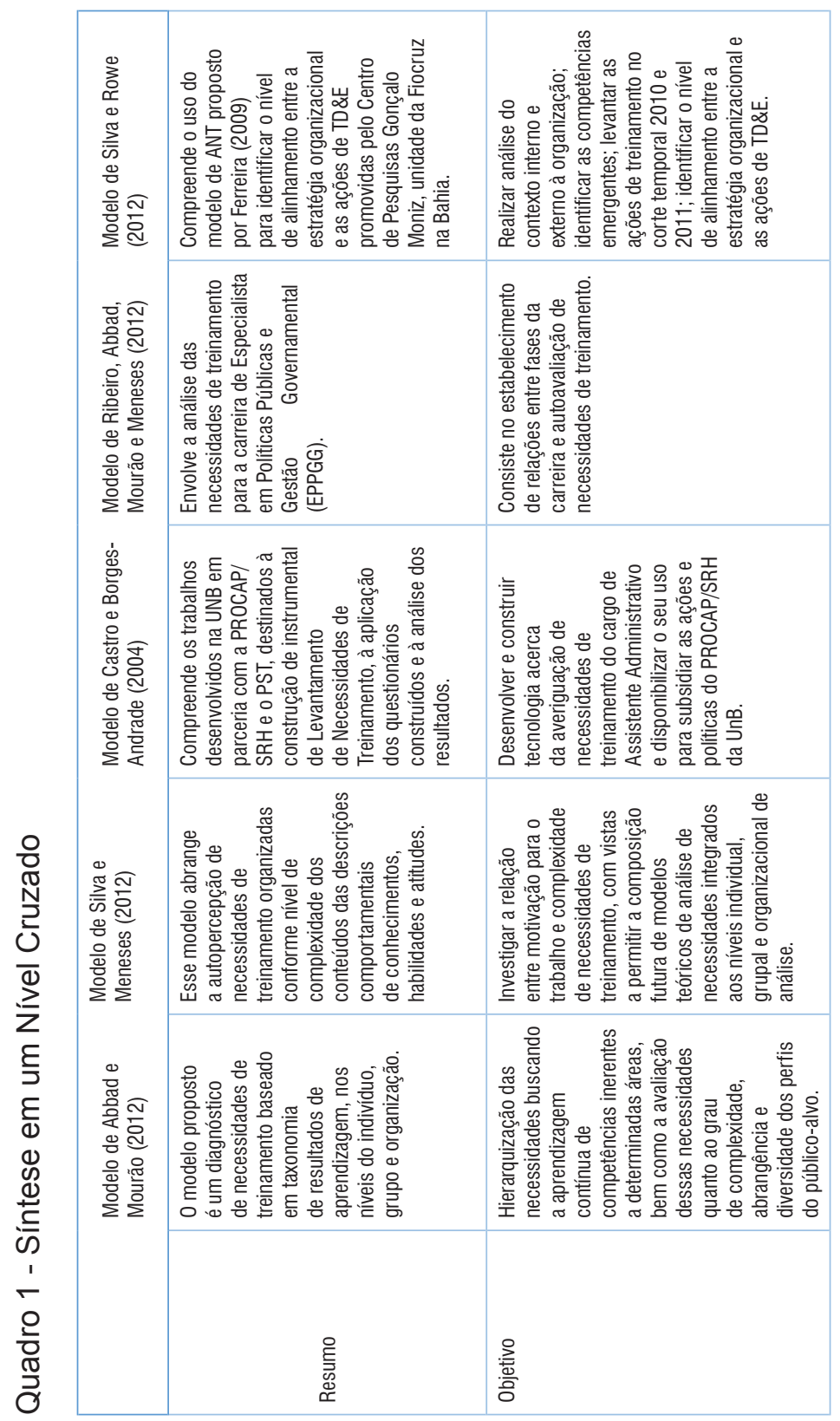




\begin{tabular}{|c|c|c|}
\hline 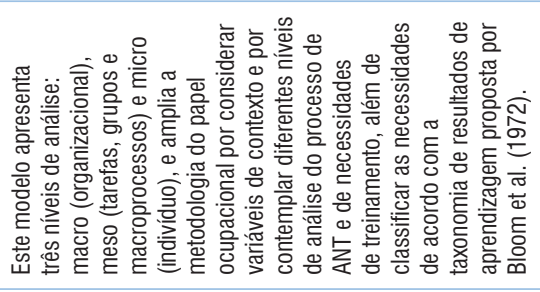 & 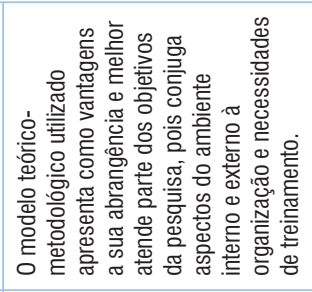 & 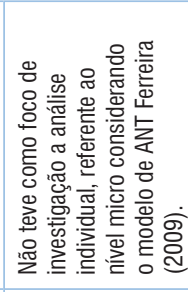 \\
\hline 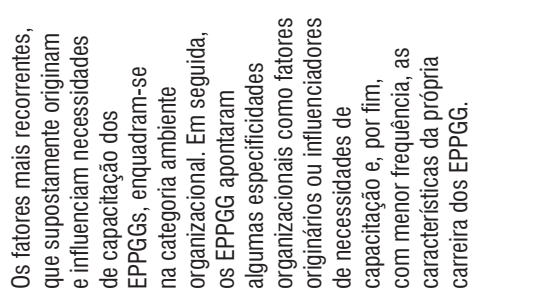 & 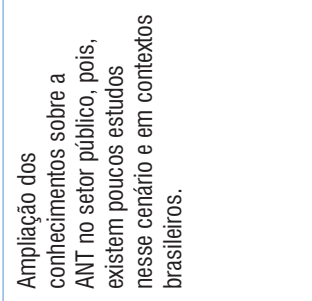 & 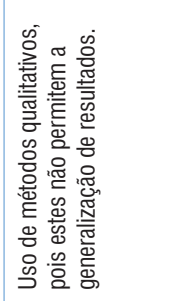 \\
\hline 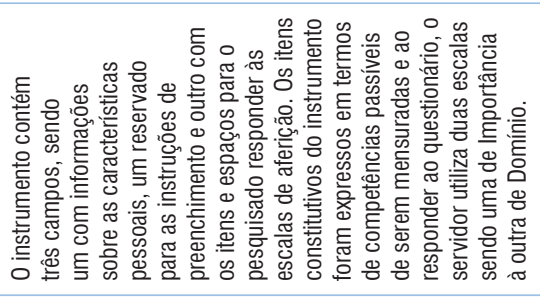 & 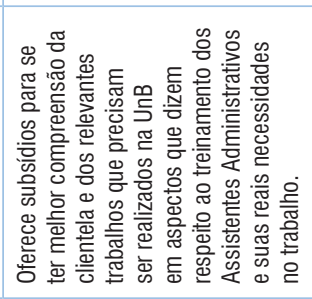 & 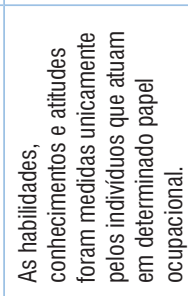 \\
\hline 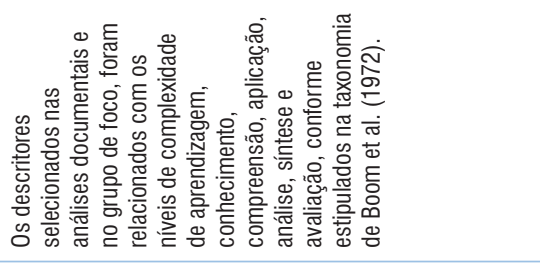 & 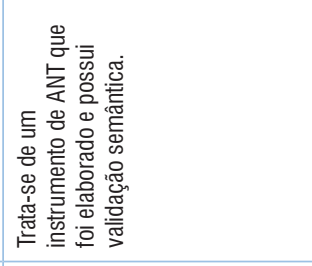 & 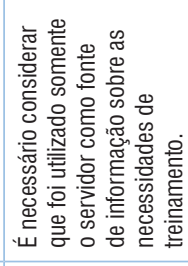 \\
\hline 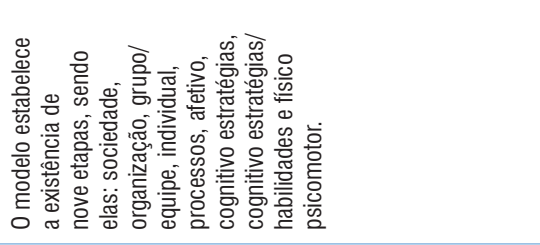 & 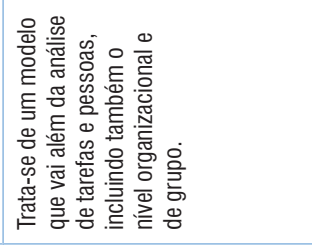 & 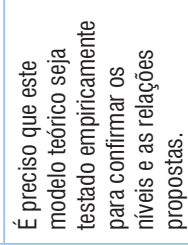 \\
\hline 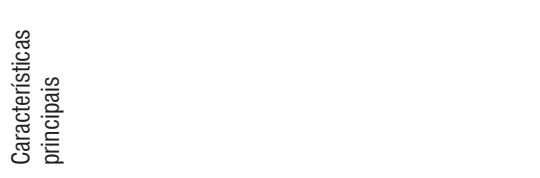 & 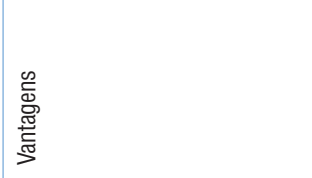 & 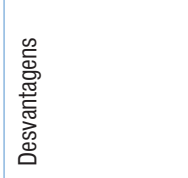 \\
\hline
\end{tabular}




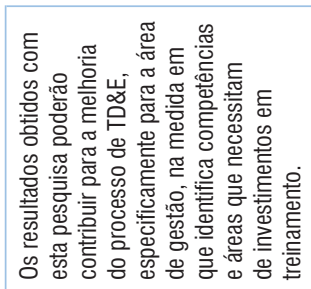

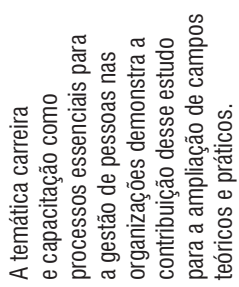

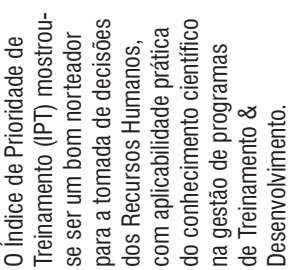
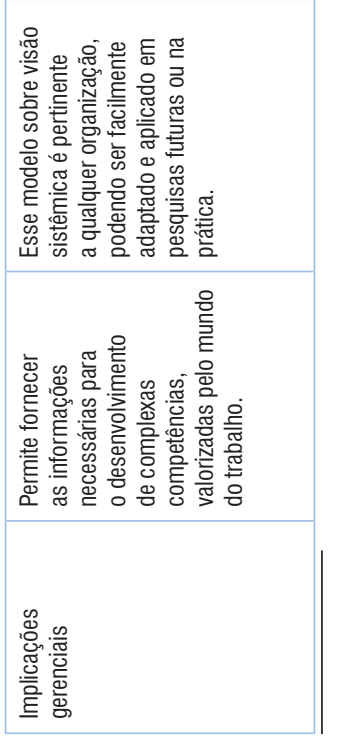

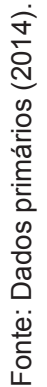

Rev. Ciênc. Admin., Fortaleza, v. 21, n. 2, p. 365-388, jul./dez. 2015 


\subsection{Construção da teoria a partir da metassíntese}

O tipo de análise de necessidades de treinamento denominado como Levantamento de Necessidades de Treinamento está associado à aplicação de cursos pré-determinados. Durante análise dos modelos e artigos, foi possível perceber que o uso da expressão "Análise de Necessidades de Treinamento" é utilizado quando se trata de pesquisas teóricas e metodológicas consistentes.

Os modelos analisados utilizam o conceito de desenvolvimento de competências, previsto na literatura como a capacidade que a pessoa possui em lidar com fatos imprevistos. Assim, as organizações e os indivíduos que nela se encontram precisam buscar continuamente recursos para adaptar e desenvolver as competências exigidas pelo mercado. Esses modelos também consideram a importância de se determinar os conhecimentos, habilidades e atitudes (CHA) dos indivíduos, com o intuito de perceber quais as necessidades que precisam ser ponderadas nos treinamentos.

De acordo com a teoria apresentada no referencial teórico deste estudo, o diagnóstico de necessidades de treinamento considera que três grandes análises devem ser utilizadas: a análise organizacional, a análise de tarefas e a análise individual. Dessa forma, os modelos apresentados por Abbad e Mourão (2012) e Silva e Rowe (2012) utilizam essa estrutura, fazendo com que o modelo proposto possua maior abrangência, sendo possível alinhar os objetivos individuais com metas, resultados e objetivos estratégicos da organização.

Os demais modelos apresentam apenas propostas nos níveis do indivíduo e de tarefas, direcionando esforços para a identificação de indivíduos, grupos e equipes que necessitam do mapeamento e desenvolvimento de $\mathrm{CHA}$, para que possam realizar adequadamente suas atividades.

O nível de análise de tarefa procura identificar e detalhar as atribuições e responsabilidades do papel ocupacional; para isso, algumas estratégias e procedimentos são utilizados, como análise documental, 
observação participativa, entrevista e incidentes críticos. Todos os modelos analisados neste trabalho consideram que essas técnicas são necessárias para um melhor detalhamento e precisão das informações, mas a escolha das técnicas varia de acordo com os dados necessários. Essa análise do papel ocupacional foi proposta pela primeira vez por Borges-Andrade e Lima (1983) e, como pode ser observado, está sendo utilizada até hoje para analisar as necessidades de treinamento no nível de tarefas e do indivíduo.

Um instrumento utilizado durante a análise individual é o cálculo de Índice de Prioridade de Treinamento, que consiste em estabelecer indivíduos e competências com maiores prioridades de treinamento. Neste trabalho, os modelos que utilizaram esse cálculo são Modelo de Castro e Borges-Andrade (2004), Rowe (2012) e Silva e Meneses (2012). Outro aspecto relevante é considerado pelos modelos de Abbad e Mourão (2012), Silva e Meneses (2012), e Silva e Rowe (2012), que consiste na utilização da teoria da taxonomia de Bloom (1972), envolvendo os três domínios da aprendizagem, denominados psicomotor, cognitivo e afetivo.

Destarte, pode-se dizer que os modelos apresentados são pertinentes em suas proposições. Porém, os modelos expostos por Abbad e Mourão (2012) e Silva e Rowe (2012), de acordo com a teoria estudada, são os mais abrangentes, sendo capazes de articular objetivos individuais com as metas e resultados observados nos níveis de grupo, organizacional e social.

\section{Considerações finais}

O estudo apresentou como objetivo pesquisar e analisar quais os modelos de análise de necessidades de treinamento presentes na literatura nacional, publicados nos últimos onze anos, nas revistas qualificadas pela Capes como A1, A2, B1 e B2. Dessa maneira, os objetivos específicos deste trabalho consistem em analisar os principais temas, autores e modelos pesquisados em análise de necessidades de treinamento. 
De acordo com a análise realizada entre os modelos e a teoria, pode-se concluir que é viável o estabelecimento de ações de TD\&E que promovam o desenvolvimento de conhecimentos, habilidades e atitudes dos indivíduos. Sendo necessário, também, realizar uma análise ampla que abranja os níveis de análise organizacional, análise de tarefas e análise individual, como é proposto pelos modelos apresentados por Abbad e Mourão (2012) e Silva e Rowe (2012), pois desse modo é possível ter uma visão holística da organização, sendo possível articular objetivos individuais com as metas e resultados observados nos níveis de grupo, organizacional e social.

Conforme observado durante as pesquisas realizadas neste trabalho, a temática de TD\&E é conduzida principalmente por pesquisadores da área de psicologia, sendo esse um dos motivos de grande parte das pesquisas centrar-se no nível do indivíduo, pois o comportamento humano é um dos principais objetos de estudo da psicologia.

Ao realizar-se uma análise entre os modelos apresentados e a teoria sobre análise de necessidades de treinamento, pode-se perceber que esta área encontra-se estagnada, sobretudo baseando-se no nível da análise organizacional, pois poucos artigos apresentam metodologias consistentes em relação a esse nível. Cabe ainda ressaltar a escassez de pesquisa relacionada à área de administração, apesar de tal nível abarcar todo o campo de conhecimento desse curso.

Surge um questionamento: por que esse nível de análise é pouco explorado na área de administração, sendo que esta obtém um maior domínio sobre esse campo? Uma resposta pode ser o fato de o estudo ser realizado, em sua maioria, por pesquisadores da área de psicologia. Sugere-se, assim, que pesquisadores da área de administração compreendam tal estudo a partir do nível de análise organizacional, com o intuito de complementar as pesquisas já existentes.

As pesquisas analisadas mostram também que é necessário alinhar os objetivos organizacionais com os objetivos dos colaboradores. Surgem, então, outros questionamentos. Esse alinhamento de objetivos 
é possível? O real interesse das organizações consiste em atingir os objetivos organizacionais, alinhando-os aos objetivos dos indivíduos, ou em alinhar os objetivos dos indivíduos aos interesses da organização?

Para as organizações, é mais interessante e lucrativo alinhar os indivíduos aos objetivos organizacionais. Os estudos aqui exibidos apresentam essa linha de pensamento, por se tratarem de trabalhos da área de psicologia, que se centram principalmente no nível do indivíduo.

O estudo apresenta como limitações o fato de a temática de TD\&E ser conduzida principalmente por pesquisadores da área de psicologia, dificultando, assim, a coleta de dados e o desenvolvimento do objetivo inicial da pesquisa, que consistia na proposição de um novo modelo de ANT.

Considerando esta pesquisa, pode-se propor que sejam realizadas outras que visem testar os modelos ainda não validados empiricamente. Outro estudo relevante é a proposição de um novo modelo que complemente os modelos aqui analisados.

\section{Referências}

ABBAD, G. da. S.; MOURÃO, L. Avaliação de necessidades de TD\&E: proposição de um novo modelo. Revista de Administração Mackenzie. São Paulo, v. 13, n. 6, ed. Especial, p. 1-31. Nov./Dez. 2012.

CASTRO, P. M. R. de.; BORGES-ANDRADE, J. E. Identificação das necessidades de capacitação profissional: o caso dos assistentes administrativos da Universidade de Brasília. Revista de Administração da USP, São Paulo, v.39, n.1, p.96-108, jan./fev./mar. 2004.

CERVO, A. L.; BERVIAN, P. A.; SILVA, R. da. Metodologia Científica. 6. ed. São Paulo: Perarson Prentice Hall, 2007.

CHIAVENATO, I. Como transformar o RH (de um centro de despesa) em um centro de lucro. São Paulo: Makron Books, 1996. 
FERREIRA, R. R. Avaliação de Necessidade de Treinamento: proposição e aplicação de um modelo teórico-metodológico nos níveis macro e meso organizacionais. 2009. 219f. Dissertação (Mestrado em Psicologia Social, do Trabalho e das Organizações). Programa de PósGraduação em Psicologia, Universidade de Brasília. Brasília.

FLEURY, A.; FLEURY, M. T. L. Estratégias empresariais e formação de competências: um quebra cabeça caleidoscópico da indústria brasileira. 2. ed. São Paulo: Atlas, 2001.

GIL, A. C. Gestão de Pessoas. São Paulo: Atlas, 2001.

GIL, A.C. Administração de recursos humanos: um enfoque profissional. São Paulo: Atlas, 1994.

HOON, C. Meta-synthesis of qualitative case studies: an approach to the building. Organizational Research Methods, [s.I], v. 16, n. 4, p. 522-556, Oct., 2013.

MENESES, P. P. M. Avaliação de um curso de desenvolvimento regional sustentável no nível de resultados: a contribuição dos modelos lógicos e do método quase- experimental. 2007, 244f. Tese (Doutorado em Psicologia). - Departamento de Psicologia Social e do Trabalho da Universidade de Brasília, Brasília, 2007.

MENESES, P.; ZERBINI, T.; ABBAD, G. Manual de treinamento organizacional. Porto Alegre: Artmed, 2010.

MORAES, G. Desenvolvimento de um Modelo para o Levantamento de Necessidades de Treinamento e Desenvolvimento de Recursos Humanos. Dissertação (Mestrado em Engenharia de Produção). - Universidade Federal de Santa Catarina. Programa de Pós-Graduação em Engenharia de Produção. Florianópolis, 2002.

PILATI, R. História e Importância de TD\&E. In: BORGESANDRADE, J. E.; ABBAD, G. S.; MOURÃO, L. (Orgs.). Treinamento, Desenvolvimento e Educação em Organizações e Trabalho: 
fundamentos para a gestão de pessoas. Porto Alegre: Artmed, 2006. p. 159-176.

POZAS, O. H; JAUREGUI, K. L. A snapshot of training practices in Peru. Estudios Gerenciales, Cali, v. 28, n. 124, p. 67-85; july./sept. 2012.

RIBEIRO, L. A. ABBAD, G. da. S. SILVA, L. M. C. e. MENESES. P. P. M. Análise de necessidade de treinamento para a carteira de especialista em Políticas Públicas e Gestão Governamental. In: ENCONTRO ANUAL DA ASSOCIAÇÃO NACIONAL DOS PROGRAMAS DE PÓS- GRADUAÇÃO EM ADMINISTRAÇÃOENANPAD, 36, Rio de Janeiro. Anais... Rio de Janeiro: ANPAD, 2012 p. 1-16.

SANTOS. A. R. dos. Metodologia científica: a construção do conhecimento. 7. ed. Rio de Janeiro: Lamparina, 2007.

SILVA, G. G. da; MENESES, P. P. M.. Necessidades de treinamento organizacional e motivação para trabalhar. REAd. Rev. eletrôn. adm. Porto Alegre, v..18, n.1, p. 27-62, jan./abr., 2012. ISSN 1413-2311.

SILVA, G. G. Necessidades de treinamento e motivação para trabalhar: validação de escalas e testes de relacionamentos. 2010. 142f.. Dissertação (Mestrado em Administração). Programa de PósGraduação em Administração da Universidade de Brasília, Brasília, 2010.

SILVA, R. O. da. ROWE, D. E. O. Avaliação das Necessidades de Treinamento: um Estudo da área de Gestão da Fiocruz na Bahia. In: ENCONTRO ANUAL DA ASSOCIAÇÃO NACIONAL DOS PROGRAMAS DE PÓS-GRADUAÇÃO EM ADMINISTRAÇÃOENANPAD, 36, 2012, Rio de Janeiro. Anais... Rio de Janeiro: ANPAD, 2012 p. 1-16.

SKORA, C. M.; MENDES, D. As Coisas Novas: Porque TGA parou no tempo? In: ENCONTRO ANUAL DA ASSOCIAÇÃO NACIONAL 
DOS PROGRAMAS DE PÓS- GRADUAÇÃO EM ADMINISTRAÇÃOENANPAD, 25., 2001, Campinas. Anais... Campinas: ANPAD, 2001. p. 1-16. 1 CD- ROM.

\section{Apêndice A - Artigos analisados na presente pesquisa}

ABBAD, G. da. S.; MOURÃO, L. Avaliação de necessidades de TD\&E: proposição de um novo modelo. Revista de Administração Mackenzie. São Paulo, v. 13, n. 6, ed. Especial, p. 1-31. nov./dez. 2012.

CASTRO, P. M. R. de.; BORGES-ANDRADE, J. E. Identificação das necessidades de capacitação profissional: o caso dos assistentes administrativos da Universidade de Brasília. Revista de Administração da USP, São Paulo, v.39, n.1, p.96-108, jan./fev./mar. 2004.

RIBEIRO, L. A. ABBAD, G. da. S. SILVA, L. M. C. e. MENESES. P. P. M. Análise de necessidade de treinamento para a carteira de especialista em Políticas Públicas e Gestão Governamental. Encontro da Anpad, 36., 2012, Rio de Janeiro. Anais... Rio de Janeiro, 22 a 26 de setembro de 2012, p. 1-16. 1 CD ROM.

SILVA, G. G. da; MENESES, P. P. M.. Necessidades de treinamento organizacional e motivação para trabalhar. REAd. Rev. eletrôn. adm. Porto Alegre, v..18, n.1, p. 27-62, jan./abr., 2012. ISSN 1413-2311.

SILVA, R. O. da. ROWE, D. E. O. Avaliação das Necessidades de Treinamento: um Estudo da área de Gestão da Fiocruz na Bahia. In: ENCONTRO ANUAL DA ASSOCIAÇÃO NACIONAL DOS PROGRAMAS DE PÓS-GRADUAÇÃO EM ADMINISTRAÇÃOENANPAD, 36, 2012, Rio de Janeiro. Anais... Rio de Janeiro: ANPAD, 2012 p. 1-16.

Artigo recebido em: 05/04/2015

Aprovado em: 28/09/2015 